\title{
Maximal independent sets in bipartite graphs with at least one cycle
}

\author{
Shuchao $\mathrm{Li}^{1}$ \\ Huihui Zhang ${ }^{1}$ \\ Xiaoyan Zhang ${ }^{2 \dagger}$ \\ ${ }^{1}$ Faculty of Mathematics and Statistics, Central China Normal University, Wuhan 430079, China \\ ${ }^{2}$ School of Mathematical Science \& Institute of Mathematics, Nanjing Normal University, Nanjing 210023, China
}

received $18^{\text {th }}$ Apr. 2013, revised 30 $0^{\text {th }}$ July 2013, accepted $21^{\text {st }}$ Aug. 2013.

\begin{abstract}
A maximal independent set is an independent set that is not a proper subset of any other independent set. Liu [J.Q. Liu, Maximal independent sets of bipartite graphs, J. Graph Theory, 17 (4) (1993) 495-507] determined the largest number of maximal independent sets among all $n$-vertex bipartite graphs. The corresponding extremal graphs are forests. It is natural and interesting for us to consider this problem on bipartite graphs with cycles. Let $\mathscr{B}_{n}$ (resp. $\mathscr{B}_{n}^{\prime}$ ) be the set of all $n$-vertex bipartite graphs with at least one cycle for even (resp. odd) $n$. In this paper, the largest number of maximal independent sets of graphs in $\mathscr{B}_{n}$ (resp. $\mathscr{B}_{n}^{\prime}$ ) is considered. Among $\mathscr{B}_{n}$ the disconnected graphs with the first-, second-, $\ldots, \frac{n-2}{2}$-th largest number of maximal independent sets are characterized, while the connected graphs in $\mathscr{B}_{n}$ having the largest and the second largest number of maximal independent sets are determined. Among $\mathscr{B}_{n}^{\prime}$ graphs having the largest number of maximal independent sets are identified.
\end{abstract}

Keywords: Maximal independent set, bipartite graph, cycle

\section{Introduction}

Given a graph $G=\left(V_{G}, E_{G}\right)$, a set $I \subseteq V_{G}$ is independent if there is no edge of $G$ between any two vertices of $I$. A maximal independent set is an independent set that is not a proper subset of any other independent set. The dual of an independent set is a clique, in the sense that a clique corresponds to an independent set in the complement graph. The set of all maximal independent sets of a graph $G$ is denoted by $\operatorname{MI}(G)$ and its cardinality by $\operatorname{mi}(G)$.

Around 1960, Erdős and Moser proposed the problem to determine the maximum value of $\operatorname{mi}(G)$ when $G$ runs over all $n$-vertex graphs and to characterize the graphs attaining this maximum, both of which were answered by Moon and Moser [18]. It is interesting to see that the extremal graphs turn to have most components isomorphic to the complete graph $K_{3}$. On the other hand, the theory on maximal independent set has some applications in other research field. For example, in chemistry, a Clar structure is defined to be a maximal independent set of vertices of the Clar graph of the corresponding benzenoid hydrocarbons [5]. Clar structures recently are used as basis-set to compute resonance energies. The theory of maximal

\footnotetext{
$\dagger$ Email: royxyzhang@gmail.com.

1365-8050 @ 2013 Discrete Mathematics and Theoretical Computer Science (DMTCS), Nancy, France
} 
independent set is also applied to the areas of managements and networks. For the compatibility graph $G$, its vertices denote tasks, and an edge denotes a resource sharing constraint between the two tasks linked by it. A maximal independent set of a compatibility graph represents a maximal set of tasks that can be executed concurrently. Basagni [2] and Alzoubi et al. [21] pointed out the importance of a maximal independent set in the wireless network. Moscibroda and Watenhofer [19] obtained the wellknown Maximal Independent Set problem when they modeled the unstructured radio network as a graph.

Along the line on the study of the maximal independent set in mathematical literature, mathematicians focused on determining the largest number of $\operatorname{mi}(G)$ in various interesting classes of graphs. Ying et al. [22] determined the maximum number of maximal independent sets in graphs of order $n$ with at most $r$ cycles and in connected graphs of order $n \geqslant 3 r$ with at most $r$ cycles. Ortiz [20] established a sharp upper bound for the number of maximal independent sets in caterpillar graphs. Arumugam et al. [1] studied the maximal independent set of graphs with minimum coloring. Duffus, Frankl and Rödl [4] studied maximal independent sets in graphs obtained from Boolean lattices. Jing and one of the present authors [16] studied the maximal independent set of graphs with cyclomatic number at most two. Jin and Li [8] determined the second largest number of maximal independent sets among all graphs of order $n$. Hua and Hou [6] determined the $n$-vertex graph having the third largest number of maximal independent sets. Jou and Lin $[10,13]$ settled the problem for trees and forests. Jin and Yan [9] settled the problem for the second and third largest number of maximal independent sets of trees.

This paper is motivated directly from [17], in which the author completely characterized the $n$-vertex bipartite graphs having the largest number of maximal independent sets. The corresponding extremal graphs are forests. Furthermore, for the bipartite graph of order $n$ that contains cycles, the author in [17] determined the upper bound on the number of maximal independent sets for odd $n$. Unfortunately, the graphs which attain this value were not characterized. It is natural and interesting to determine the corresponding extremal graphs, which is settled in this paper. On the other hand, it is necessary and interesting to consider this problem on the $n$-vertex bipartite graphs with cycles for even $n$. In this paper, among the set of all disconnected bipartite graphs with even order, the extremal graphs which have the first-, second-, $\ldots, \frac{n-2}{2}$-th largest number of maximal independent sets are characterized respectively, while among the set of all connected bipartite graphs with even order, the extremal graphs having the largest and the second largest number of maximal independent sets are identified.

\section{Preliminary}

Given a simple graph $G=\left(V_{G}, E_{G}\right)$, the cardinality of $V_{G}$ is called the order of $G$. And $G-v$ denotes the graph obtained from $G$ by deleting vertex $v \in V_{G}$ (this notation is naturally extended if more than one vertex is deleted). For $v \in V_{G}$, let $N_{G}(v)$ (or $N(v)$ for short) denote the set of all the adjacent vertices of $v$ in $G$ and $d(v)=\left|N_{G}(v)\right|$. For convenience, let $N_{G}[v]=N_{G}(v) \cup\{v\}$. A leaf of $G$ is a vertex of degree one, while an isolated vertex of $G$ is a vertex of degree zero. For any two graphs $G$ and $H$, let $G \uplus H$ denote the disjoint union of $G$ and $H$, and for any nonnegative integer $t$, let $t G$ stand for the disjoint union of $t$ copies of $G$. For a connected graph $H$ with maximum degree vertex $x$ and a graph $G=G_{1} \uplus G_{2} \uplus \cdots \uplus G_{k}$ with $u_{i}$ being a maximum degree vertex in $G_{i}, i=1,2, \ldots, k$. Define the graph $H * G$ to be the graph with vertex set $V_{H * G}=V_{H} \cup V_{G}$ and edge set $E_{H * G}=E_{H} \cup E_{G} \cup\left\{x u_{i}: i=1,2, \ldots, k\right\}$. An odd (resp. even) component is a component of odd (resp. even) order. Throughout the text we denote by $P_{n}, C_{n}, K_{1, n-1}$ and $K_{n}$ the path, cycle, star and complete graph on $n$ vertices, respectively. Undefined terminology and notation may be referred to [3]. 
Throughout this paper, for simplicity, $r$ denotes $\sqrt{2}$.

We begin with some useful known results which are needed to prove our main results.

Lemma $1([7,14])$ For any vertex $v$ in a graph $G, \operatorname{mi}(G) \leqslant \operatorname{mi}(G-v)+\operatorname{mi}\left(G-N_{G}[v]\right)$. If $v$ is a leaf adjacent to $u$, then $\operatorname{mi}(G)=\operatorname{mi}\left(G-N_{G}[v]\right)+\operatorname{mi}\left(G-N_{G}[u]\right)$.

Lemma 2 ([14]) If $G$ is the union of two disjoint graphs $G_{1}$ and $G_{2}$, then $\operatorname{mi}(G)=\operatorname{mi}\left(G_{1}\right) \cdot \operatorname{mi}\left(G_{2}\right)$.

Lemma 3 ([13]) If $T$ is a tree with $n \geqslant 1$ vertices, then $\operatorname{mi}(T) \leqslant t_{1}(n)$, where

$$
t_{1}(n)= \begin{cases}r^{n-2}+1, & \text { if } n \text { is even } \\ r^{n-1}, & \text { if } n \text { is odd }\end{cases}
$$

Furthermore, $\operatorname{mi}(T)=t_{1}(n)$ if and only if $T \in T_{1}(n)$, where

$$
T_{1}(n)= \begin{cases}B\left(2, \frac{n-2}{2}\right) \text { or } B\left(4, \frac{n-4}{2}\right), & \text { if } n \text { is even; } \\ B\left(1, \frac{n-1}{2}\right), & \text { if } n \text { is odd }\end{cases}
$$

where $B(i, j)$ is the set of batons, which are the graphs obtained from a basic path $P_{i}(i \geqslant 1)$ by attaching $j \geqslant 0$ paths of length two to the endpoints of $P_{i}$ in any possible ways.

Lemma 4 ([13]) If $F$ is a forest with $n \geqslant 1$ vertices, then $\operatorname{mi}(F) \leqslant f_{1}(n)$, where

$$
f_{1}(n)= \begin{cases}r^{n}, & \text { if } n \text { is even } \\ r^{n-1}, & \text { if } n \text { is odd }\end{cases}
$$

Furthermore, $\operatorname{mi}(F)=f_{1}(n)$ if and only if $F \in F_{1}(n)$, where

$$
F_{1}(n)= \begin{cases}\frac{n}{2} K_{2}, & \text { if } n \text { is even; } \\ B\left(1, \frac{n-1-2 s}{2}\right) \uplus s K_{2} \text { for some s with } 0 \leqslant s \leqslant \frac{n-1}{2}, & \text { if } n \text { is odd. }\end{cases}
$$

Lemma 5 ([14]) If $F$ is a forest with $n \geqslant 4$ vertices having $F \notin F_{1}(n)$, then $\operatorname{mi}(F) \leqslant f_{2}(n)$, where

$$
f_{2}(n)= \begin{cases}3 r^{n-4}, & \text { if } n \geqslant 4 \text { is even } \\ 3, & \text { if } n=5 \\ 7 r^{n-7}, & \text { if } n \geqslant 7 \text { is odd }\end{cases}
$$

Furthermore, $\operatorname{mi}(F)=f_{2}(n)$ if and only if $F \in F_{2}(n)$, where

$$
F_{2}(n)= \begin{cases}P_{4} \uplus \frac{n-4}{2} K_{2}, & \text { if } n \geqslant 4 \text { is even; } \\ P_{1} * P_{4} \text { or, } P_{1} \uplus P_{4}, & \text { if } n=5 ; \\ P_{7} \uplus \frac{n-7}{2} K_{2}, & \text { if } n \geqslant 7 \text { is odd. }\end{cases}
$$


For $n \geqslant 2,0 \leqslant k \leqslant\left\lfloor\frac{n}{2}\right\rfloor$, define

$$
B_{n, k}= \begin{cases}2 P_{4} \uplus \frac{n-8}{2} K_{2} \text { or, } T_{1}(n-2 k) \uplus k K_{2}, & \text { if } n \text { is even; } \\ T_{1}(n-2 k) \uplus k K_{2}, & \text { if } n \text { is odd. }\end{cases}
$$

Then $\operatorname{mi}\left(B_{n, k}\right)=2^{k} \cdot \operatorname{mi}\left(T_{1}(n-2 k)\right)$, or $9 r^{n-8}$.

Lemma 6 ([17]) The maximum number of maximal independent sets among all bipartite graphs of order $n$ is $2^{\left\lfloor\frac{n}{2}\right\rfloor}$, and the only bipartite graphs of order $n$ which have this many maximal independent sets are $F_{1}(n)$.

Lemma 7 ([17]) If $G$ is an acyclic graph of order $n$ and $G \not B_{n, k}$, then $\operatorname{mi}(G)<t_{1}(n)$.

Lemma 8 If $n \geqslant 6$, then $\operatorname{mi}\left(C_{n}\right)=\operatorname{mi}\left(C_{n-2}\right)+\operatorname{mi}\left(C_{n-3}\right)$. Furthermore, one has

$$
\operatorname{mi}\left(C_{n}\right)< \begin{cases}r^{n-1}, & \text { if } n \geqslant 7 \text { is odd } \\ r^{n-2}, & \text { if } n \geqslant 12 \text { is even. }\end{cases}
$$

Proof: The first part is due to [13]; we show the second part by induction on $n$. It is routine to check that

$$
\begin{aligned}
\operatorname{mi}\left(C_{7}\right) & =7<r^{6}, & \operatorname{mi}\left(C_{9}\right) & =12<r^{8}, \\
\operatorname{mi}\left(C_{12}\right) & =29<r^{10}, & \operatorname{mi}\left(C_{11}\right) & =22<r^{10}, \\
\operatorname{mi}\left(C_{13}\right) & =39<r^{12}, & \operatorname{mi}\left(C_{14}\right) & =51<r^{12} .
\end{aligned}
$$

Assume the result holds for $n \leqslant k$. Now consider $n=k+1 \geqslant 15$. By induction hypothesis, we obtain

$$
\operatorname{mi}\left(C_{n}\right)=\operatorname{mi}\left(C_{n-2}\right)+\operatorname{mi}\left(C_{n-3}\right)< \begin{cases}r^{n-2-1}+r^{n-3-2}=3 r^{n-5}<r^{n-1}, & \text { if } n \text { is odd; } \\ r^{n-2-2}+r^{n-3-1}=r^{n-2}, & \text { if } n \text { is even. }\end{cases}
$$

This completes the proof.

\section{Sharp bounds and extremal graphs}

Let $\mathscr{B}_{n}$ (resp. $\mathscr{B}_{n}^{\prime}$ ) be the set of all $n$-vertex bipartite graphs with at least one cycle for even (resp. odd) $n$. In this section, among $\mathscr{B}_{n}$ the disconnected graphs with the first-, second-, $\ldots, \frac{n-2}{2}$-th largest number of maximal independent sets are characterized, while the connected graphs in $\mathscr{B}_{n}$ having the largest and the second largest number of maximal independent sets are determined; among $\mathscr{B}_{n}^{\prime}$ the graphs having the largest number of maximal independent sets are identified.

For even $n$ and $3 \leqslant k \leqslant \frac{n}{2}$, define $D_{n, k}=B_{2 k} \uplus\left(\frac{n}{2}-k\right) K_{2}$, where $B_{2 k}$ is depicted in Fig. 1 . Then $\operatorname{mi}\left(D_{n, k}\right)=2^{\frac{n}{2}-k} \cdot \operatorname{mi}\left(B_{2 k}\right)=2^{\frac{n}{2}-k}\left(2^{k-1}+1\right) \leqslant r^{n-2}+r^{n-6}$.

Theorem 1 For graphs $D_{n, 3}, D_{n, 4}, \ldots, D_{n, \frac{n}{2}}, C_{8} \uplus \frac{n-8}{2} K_{2}, C_{10} \uplus \frac{n-10}{2} K_{2},\left(C_{8} * K_{2}\right) \uplus \frac{n-10}{2} K_{2}$, one has

$$
\begin{aligned}
\operatorname{mi}\left(D_{n, 3}\right) & =\operatorname{mi}\left(C_{8} \uplus \frac{n-8}{2} K_{2}\right)>\operatorname{mi}\left(D_{n, 4}\right)>\operatorname{mi}\left(D_{n, 5}\right)=\operatorname{mi}\left(C_{10} \uplus \frac{n-10}{2} K_{2}\right) \\
& =\operatorname{mi}\left(\left(C_{8} * K_{2}\right) \uplus \frac{n-10}{2} K_{2}\right)>\operatorname{mi}\left(D_{n, 6}\right)>\operatorname{mi}\left(D_{n, 7}\right)>\cdots>\operatorname{mi}\left(D_{n, \frac{n}{2}}\right)=r^{n-2}+1 .
\end{aligned}
$$




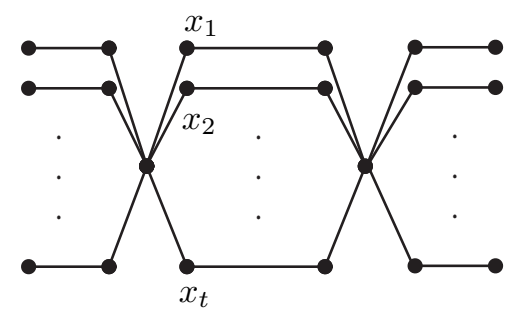

Fig. 1: Graph $B_{2 k}$, where $k \geqslant 3$ and $t \geqslant 2$.

Proof: By direct computing, we have

$$
\begin{aligned}
\operatorname{mi}\left(D_{n, t}\right) & =2^{\frac{n-2 t}{2}} \cdot \operatorname{mi}\left(B_{2 t}\right)=2^{\frac{n-2}{2}}+2^{\frac{n-2 t}{2}}, \quad t=3,4, \ldots, \frac{n}{2}, \\
\operatorname{mi}\left(C_{8} \uplus \frac{n-8}{2} K_{2}\right) & =10 \cdot 2^{\frac{n-8}{2}}, \\
\operatorname{mi}\left(C_{10} \uplus \frac{n-10}{2} K_{2}\right) & =17 \cdot 2^{\frac{n-10}{2}}, \\
\operatorname{mi}\left(\left(C_{8} * K_{2}\right) \uplus \frac{n-10}{2} K_{2}\right) & =17 \cdot 2^{\frac{n-10}{2}} .
\end{aligned}
$$

By (1), it is easy to see that $\operatorname{mi}\left(D_{n, t}\right)<\operatorname{mi}\left(D_{n, t-1}\right)$ for $4 \leqslant t \leqslant \frac{n}{2}$. Combining with (2)-(4), our results follow immediately.

Let $H_{0}(6), H_{0}(8), H_{0}(10), H_{1}(n), H_{2}(n), H_{3}(n)$ and $O_{1}(n)$ be the graphs depicted in Fig. 2.

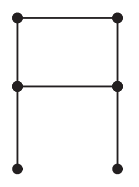

$H_{0}(6)$

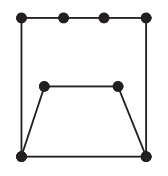

$H_{0}(8)$

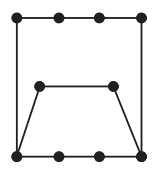

$H_{0}(10)$

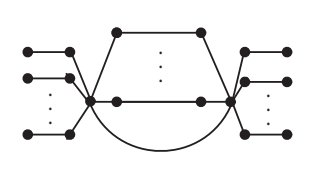

$H_{1}(n)$

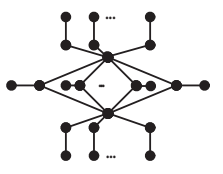

$H_{2}(n)$

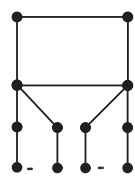

$H_{3}(n)$

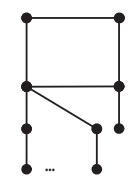

$O_{1}(n)$

Fig. 2: The extremal graphs

Theorem 2 Consider an n-vertex bipartite graph $G$ containing cycles with $n \geqslant 5$.

(i) If $G \in \mathscr{B}_{n} \backslash\left\{D_{n, 3}, D_{n, 4}, \ldots, D_{n, \frac{n}{2}}, C_{8} \uplus \frac{n-8}{2} K_{2}, C_{10} \uplus \frac{n-10}{2} K_{2},\left(C_{8} * K_{2}\right) \uplus \frac{n-10}{2} K_{2}\right\}$, then

$$
\operatorname{mi}(G) \leqslant r^{n-2} .
$$

If $G$ is connected, the equality holds in (5) if and only if $G \cong H_{0}(n), H_{1}(n), H_{2}(n)$, or $H_{3}(n)$ for $n=6,8,10 ;$ and $G \cong H_{1}(n), H_{2}(n)$, or $H_{3}(n)$ for $n \geqslant 12$.

If $G$ is disconnected, the equality holds in (5) if and only if $G \cong C_{4} \uplus \frac{n-4}{2} K_{2}, H_{0}(6) \uplus \frac{n-6}{2} K_{2}, H_{0}(8) \uplus$ $\frac{n-8}{2} K_{2}, H_{0}(10) \uplus \frac{n-10}{2} K_{2}, H_{1}(n-2 k) \uplus k K_{2}, H_{2}(n-2 k) \uplus k K_{2}$, or $H_{3}(n-2 k) \uplus k K_{2}$. 
(ii) If $G \in \mathscr{B}_{n}^{\prime}$, then

$$
\operatorname{mi}(G) \leqslant 3 r^{n-5} \text {. }
$$

The equality holds in (6) if and only if $G \cong C_{6} * K_{1}$, or $O_{1}(n)$ for connected $G$ and $G \cong O_{1}(n-$ $2 k) \uplus k K_{2}$, or $\left(C_{6} * K_{1}\right) \uplus \frac{n-7}{2} K_{2}$ for disconnected $G$.

Proof: We put the proofs of (i) and (ii) together as below. Choose $G$ in $\mathscr{B}_{n} \backslash\left\{D_{n, 3}, D_{n, 4}, \ldots, D_{n, \frac{n}{2}}\right.$, $\left.C_{8} \uplus \frac{n-8}{2} K_{2}, C_{10} \uplus \frac{n-10}{2} K_{2},\left(C_{8} * K_{2}\right) \uplus \frac{n-10}{2} K_{2}\right\}$ (resp. $\mathscr{B}_{n}^{\prime}$ ) such that $\operatorname{mi}(G)$ is as large as possible. We proceed by induction on $n$.

For $5 \leqslant n \leqslant 6$, it is straightforward to check that our results hold. Assume that our results hold for $n \leqslant k$. Now consider $n=k+1 \geqslant 7$.

First we consider that $G$ is disconnected. Denote

$$
G=G_{o_{1}} \uplus G_{o_{2}} \uplus \cdots \uplus G_{o_{1}} \uplus G_{e_{1}} \uplus G_{e_{2}} \uplus \cdots \uplus G_{e_{l_{2}}},
$$

where $G_{o_{i}}$ is an odd component for $1 \leqslant i \leqslant l_{1}$ and $G_{e_{j}}$ is an even component for $1 \leqslant j \leqslant l_{2}$.

Case 1. $n$ is even. In this case, we have that $l_{1}$ is also even. If $l_{1} \geqslant 2$, then

$$
\begin{aligned}
\operatorname{mi}(G) & =\prod_{i=1}^{l_{1}} \operatorname{mi}\left(G_{o_{i}}\right) \cdot \prod_{j=1}^{l_{2}} \operatorname{mi}\left(G_{e_{j}}\right) \quad \text { (by Lemma 2) } \\
& \leqslant \prod_{i=1}^{l_{1}} r^{\left|V_{G_{o_{i}}}\right|-1} \cdot \prod_{j=1}^{l_{2}} r^{\left|V_{G_{e_{j}}}\right|} \quad \text { (by Lemma 6) } \\
& =r^{n-l_{1}} \\
& \leqslant r^{n-2} .
\end{aligned}
$$

The equality in (7) holds if and only if $G_{o_{i}} \cong F_{1}\left(\left|V_{G_{o_{i}}}\right|\right)$ for $1 \leqslant i \leqslant l_{1}, G_{e_{j}} \cong F_{1}\left(\left|V_{G_{e_{j}}}\right|\right)$ for $1 \leqslant j \leqslant l_{2}$; while the equality in (8) holds if and only if $l_{1}=2$, which implies that $G \cong F_{1}\left(\left|V_{G_{o_{1}}}\right|\right) \uplus$ $F_{1}\left(\left|V_{G_{o_{2}}}\right|\right) \uplus \frac{n-\left|V_{G_{o_{1}}}\right|-\left|V_{G_{o_{2}}}\right|}{2} K_{2}$. Obviously, $G$ does not contain cycles, so $\operatorname{mi}(G)<r^{n-2}$.

Therefore we consider that $l_{1}=0$, that is to say, each component contained in $G$ is an even component. Without loss of generality, assume that $\left|V_{G_{e_{1}}}\right| \geqslant\left|V_{G_{e_{2}}}\right| \geqslant \cdots \geqslant\left|V_{G_{e_{2}}}\right|$. Note that $G$ contains cycles, hence $\left|V_{G_{e_{1}}}\right| \geqslant 4$.

If $\left|V_{G_{e_{1}}}\right|=4$, then there exists a $p \in\left\{1,2, \ldots, l_{2}\right\}$ such that $\left|V_{G_{e_{1}}}\right|=\left|V_{G_{e_{2}}}\right|=\left|V_{G_{e_{3}}}\right|=\cdots=$ $\left|V_{G_{e_{p}}}\right|=4$. As $G$ contains cycles, there is at least one $i \in\{1,2, \ldots, p\}$ such that $G_{e_{i}} \cong C_{4}$. We obtain that

$$
\begin{aligned}
\operatorname{mi}(G) & =\operatorname{mi}\left(G_{e_{i}}\right) \cdot \prod_{1 \leqslant j \neq i \leqslant p} \operatorname{mi}\left(G_{e_{j}}\right) \cdot \prod_{k=p+1}^{l_{2}} \operatorname{mi}\left(G_{e_{k}}\right) \quad \text { (by Lemma 2) } \\
& \leqslant 2 \cdot 3^{p-1} \cdot r^{n-4 p} \quad \text { (by Lemmas } 3 \text { and 6) } \\
& =r^{n-2} \cdot\left(\frac{3}{4}\right)^{p-1} \\
& \leqslant r^{n-2} .
\end{aligned}
$$


The equality in (9) holds if and only if $G_{e_{i}} \cong C_{4}, G_{e_{j}} \cong P_{4}$ for $1 \leqslant j \neq i \leqslant p, G-\left(G_{e_{1}} \uplus G_{e_{2}} \uplus \cdots \uplus\right.$ $\left.G_{e_{p}}\right) \cong F_{1}(n-4 p)=\frac{n-4 p}{2} K_{2}$; while the equality in (10) holds if and only if $p=1$, which implies that $G \cong C_{4} \uplus \frac{n-4}{2} K_{2}$, our result holds.

If $\left|V_{G_{e_{1}}}\right| \geqslant 6$, then we consider the following two possible subcases.

- $\left|V_{G_{e_{2}}}\right|=2$. In this subcase, we have $G-G_{e_{1}} \cong \frac{n-\left|V_{G_{e_{1}}}\right|}{2} K_{2}$. Note that $G \supsetneqq D_{n,\left|V_{G_{e_{1}}}\right| / 2}, C_{8} \uplus$ $\frac{n-8}{2} K_{2}, C_{10} \uplus \frac{n-10}{2} K_{2}$ and $\left(C_{8} * K_{2}\right) \uplus \frac{n-10}{2} K_{2}$, hence $G_{e_{1}} ¥ B_{\mid V_{G_{e_{1}}}}, C_{8}, C_{10}$ and $C_{8} * K_{2}$. By induction hypothesis and Lemma 2, we have

$$
\begin{aligned}
\operatorname{mi}(G) & =\operatorname{mi}\left(G_{e_{1}}\right) \cdot \operatorname{mi}\left(G-G_{e_{1}}\right) \\
& \leqslant r^{\left|V_{G_{e_{1}}}\right|-2} \cdot r^{n-\left|V_{G_{e_{1}}}\right|} \\
& =r^{n-2}
\end{aligned}
$$

The equality in (11) holds if and only if $G_{e_{1}} \cong H_{0}(6), H_{0}(8), H_{0}(10), H_{1}\left(\left|V_{G_{e_{1}}}\right|\right), H_{2}\left(\left|V_{G_{e_{1}}}\right|\right)$, or $H_{3}\left(\left|V_{G_{e_{1}}}\right|\right)$. Together with $G-G_{e_{1}} \cong \frac{n-\left|V_{G_{e_{1}}}\right|}{2} K_{2}$, our result follows immediately.

- $\left|V_{G_{e_{2}}}\right| \geqslant 4$. In this subcase, we have

$$
\begin{aligned}
\operatorname{mi}(G) & =\operatorname{mi}\left(G_{e_{1}}\right) \cdot \operatorname{mi}\left(G_{e_{2}}\right) \cdot \prod_{j=3}^{l_{2}} \operatorname{mi}\left(G_{e_{j}}\right) \quad(\text { by Lemma 2) } \\
& \leqslant\left(r^{\left|V_{G_{e_{1}}}\right|-2}+1\right) \cdot\left(r^{\left|V_{G_{2}}\right|-2}+1\right) \cdot r^{n-\left|V_{G_{e_{1}}}\right|-\left|V_{G_{e_{2}}}\right|} \quad \text { (by Lemmas 3,6 and Theorem 1) } \\
& =r^{n-4}+r^{n-\left|V_{G_{e_{2}}}\right|-2}+r^{n-\left|V_{G_{1}}\right|-2}+r^{n-\left|V_{G_{e_{1}}}\right|-\left|V_{G_{e_{2}}}\right|} \\
& \leqslant r^{n-4}+r^{n-6}+r^{n-8}+r^{n-10} \\
& <r^{n-2}
\end{aligned}
$$

Case 2. $n$ is odd. In this case, $l_{1}$ is also odd. If $l_{1} \geqslant 3$, by Lemmas 2 and 6 , we get

$$
\operatorname{mi}(G)=\prod_{i=1}^{l_{1}} \operatorname{mi}\left(G_{o_{i}}\right) \cdot \prod_{j=1}^{l_{2}} \operatorname{mi}\left(G_{e_{j}}\right) \leqslant \prod_{i=1}^{l_{1}} r^{\left|V_{G_{o_{i}}}\right|-1} \cdot \prod_{j=1}^{l_{2}} r^{\left|V_{G_{e_{j}}}\right|}=r^{n-l_{1}} \leqslant r^{n-3}<3 r^{n-5} .
$$

Hence, we consider that $l_{1}=1$ in what follows.

If $G_{o_{1}}$ contains cycles, by induction hypothesis and Lemmas 2 and 6 , we obtain that

$$
\operatorname{mi}(G)=\operatorname{mi}\left(G_{O_{1}}\right) \cdot \operatorname{mi}\left(G-G_{o_{1}}\right) \leqslant 3 r^{\left|V_{G_{o_{1}}}\right|-5} \cdot r^{n-\left|V_{G_{O_{1}}}\right|}=3 r^{n-5}
$$

the equality holds if and only if $G_{o_{1}} \cong O_{1}\left(\left|V_{G_{o_{1}}}\right|\right)$ or $C_{6} * K_{1}$ and $G-G_{o_{1}}=\frac{n-\left|V_{G_{o_{1}}}\right|}{2} K_{2}$, which implies that $G \cong O_{1}\left(\left|V_{G_{o_{1}}}\right|\right) \uplus \frac{n-\left|V_{G_{o_{1}}}\right|}{2} K_{2}$ or $\left(C_{6} * K_{1}\right) \uplus \frac{n-7}{2} K_{2}$, the result holds.

If $G_{o_{1}}$ does not contain cycles, then there exists at least one even component $G_{e_{j}}$ containing cycles 
with $\left|V_{G_{e_{j}}}\right| \geqslant 4$. If $\left|V_{G_{e_{j}}}\right|=4$, then $G_{e_{j}} \cong C_{4}$. We obtain that

$$
\begin{array}{rlr}
\operatorname{mi}(G) & =\operatorname{mi}\left(G_{o_{1}}\right) \cdot \operatorname{mi}\left(G_{e_{j}}\right) \cdot \prod_{1 \leqslant i \neq j \leqslant l_{2}} \operatorname{mi}\left(G_{e_{i}}\right) & \text { (by Lemma 2) } \\
& \leqslant r^{\left|V_{G_{o_{1}}}\right|-1} \cdot 2 \cdot r^{n-4-\left|V_{G_{o_{1}}}\right|} & \\
& <3 r^{n-5}, & \text { (by Lemmas 3 and 6) }
\end{array}
$$

the result holds. If $\left|V_{G_{e_{j}}}\right| \geqslant 6$, we obtain that

$$
\begin{array}{rlr}
\operatorname{mi}(G) & =\operatorname{mi}\left(G_{o_{1}}\right) \cdot \operatorname{mi}\left(G_{e_{j}}\right) \cdot \prod_{1 \leqslant i \neq j \leqslant l_{2}} \operatorname{mi}\left(G_{e_{i}}\right) & \text { (by Lemma 2) } \\
& \leqslant r^{\left|V_{G_{o_{1}}}\right|-1} \cdot\left(r^{\left|V_{G_{e_{j}}}\right|-2}+1\right) \cdot r^{n-\left|V_{G_{o_{1}}}\right|-\left|V_{G_{e_{j}}}\right|} \quad & \text { (by Lemmas 3, 6 and Theorem 1) } \\
& =r^{n-3}+r^{n-\left|V_{G_{e_{j}}}\right|-1} \\
& \leqslant r^{n-3}+r^{n-7} \\
& <3 r^{n-5} .
\end{array}
$$

The result holds.

Now we consider that $G$ is connected. It suffices to consider the following two possible cases.

Case 1. $G$ has a vertex $v$ of degree $\geqslant 4$ such that $G-v$ has cycles.

Subcase 1.1. $n$ is even. In this subcase, we have

$$
\begin{array}{rlr}
\operatorname{mi}(G) & \leqslant \operatorname{mi}(G-v)+\operatorname{mi}\left(G-N_{G}[v]\right) \quad \text { (by Lemma 1) } \\
& \leqslant 3 r^{n-6}+r^{n-5-1} \quad \text { (by induction hypothesis and Lemma 6) } \\
& =r^{n-2}
\end{array}
$$

The equality in (12) holds if and only if $G-v \cong O_{1}(k) \uplus \frac{n-k-1}{2} K_{2}$ or $\left(C_{6} * K_{1}\right) \uplus \frac{n-8}{2} K_{2}, G-N_{G}[v] \cong$ $F_{1}(n-5)$. But there is no such bipartite graph since $G-N_{G}[v]$ is obtained from $G-v$ by deleting four independent vertices, hence $\operatorname{mi}(G)<r^{n-2}$.

Subcase 1.2. $n$ is odd.

In this subcase, we first consider that $G-v \cong D_{n-1, k}$. Then by Theorem $1, \operatorname{mi}(G-v) \leqslant r^{n-3}+r^{n-7}$. If $d(v)=4$, then $G-N_{G}[v]$ is either an acyclic bipartite graph which is not isomorphic to $B_{n-5, k}$ or a bipartite graph with cycles which is not isomorphic to $D_{n-5, k}$. Hence by induction hypothesis or Lemma $7, \operatorname{mi}\left(G-N_{G}[v]\right) \leqslant r^{n-7}$, which gives

$$
\begin{array}{rlr}
\operatorname{mi}(G) & \left.\leqslant \operatorname{mi}(G-v)+\operatorname{mi}\left(G-N_{G}[v]\right) \quad \quad \text { (by Lemma } 1\right) \\
& \leqslant r^{n-3}+r^{n-7}+r^{n-7} \\
& =3 r^{n-5}
\end{array}
$$

The equality in (13) holds if and only if $G-v \cong C_{6} \uplus \frac{n-7}{2} K_{2}$ and $G-N_{G}[v] \cong H_{0}(6) \uplus \frac{n-11}{2} K_{2}, H_{0}(8) \uplus$ $\frac{n-13}{2} K_{2}, H_{0}(10) \uplus \frac{n-15}{2} K_{2}, H_{1}(k) \uplus \frac{n-k-5}{2} K_{2}, H_{2}(k) \uplus \frac{n-k-5}{2} K_{2}$, or $H_{3}(k) \uplus \frac{n-k-5}{2} K_{2}$. Note that 
$G-v \cong C_{6} \uplus \frac{n-7}{2} K_{2}$, hence $G-N_{G}[v]$ is a subgraph of $P_{5} \uplus \frac{n-7}{2} K_{1}$. It is easy to see that $G-N_{G}[v]$ contains no cycles, a contradiction.

Now we consider that $G-v \nsucceq D_{n-1, k}$. In order to use the induction hypothesis, we should show that $G-v \nsucceq C_{8} \uplus \frac{n-9}{2} K_{2}, C_{10} \uplus \frac{n-11}{2} K_{2}$, and $\left(C_{8} * K_{2}\right) \uplus \frac{n-11}{2} K_{2}$ first. In fact, if $G-v \cong C_{8} \uplus \frac{n-9}{2} K_{2}$, then $1 \leqslant\left|N_{G}(v) \cap N_{C_{8}}(v)\right| \leqslant 4$.

If $\left|N_{G}(v) \cap N_{C_{8}}(v)\right|=1$, then $n \geqslant 15$ and $G-N_{G}[v] \cong P_{7} \uplus \frac{n-9}{2} K_{1}$. By Lemma 1, we get $\operatorname{mi}(G) \leqslant \operatorname{mi}(G-v)+\operatorname{mi}\left(G-N_{G}[v]\right)=5 r^{n-7}+7<3 r^{n-5}$.

If $\left|N_{G}(v) \cap N_{C_{8}}(v)\right|=2$, then $n \geqslant 13$ and $G-N_{G}[v] \cong P_{5} \uplus \frac{n-7}{2} K_{1}$, or $2 P_{3} \uplus \frac{n-9}{2} K_{1}$. By Lemma 1 , we get $\operatorname{mi}(G) \leqslant \operatorname{mi}(G-v)+\operatorname{mi}\left(G-N_{G}[v]\right)=5 r^{n-7}+4<3 r^{n-5}$.

If $\left|N_{G}(v) \cap N_{C_{8}}(v)\right|=3$, then $n \geqslant 11$ and $G-N_{G}[v] \cong P_{3} \uplus \frac{n-5}{2} K_{1}$. By Lemma 1, we get $\operatorname{mi}(G) \leqslant \operatorname{mi}(G-v)+\operatorname{mi}\left(G-N_{G}[v]\right)=5 r^{n-7}+2<3 r^{n-5}$.

If $\left|N_{G}(v) \cap N_{C_{8}}(v)\right|=4$, then $n \geqslant 9$ and $G-N_{G}[v] \cong \frac{n-1}{2} K_{1}$. By Lemma 1 , we get $\operatorname{mi}(G) \leqslant$ $\operatorname{mi}(G-v)+\operatorname{mi}\left(G-N_{G}[v]\right)=5 r^{n-7}+1<3 r^{n-5}$.

By a similar discussion as above, we may show that $G-v \neq C_{10} \uplus \frac{n-11}{2} K_{2},\left(C_{8} * K_{2}\right) \uplus \frac{n-11}{2} K_{2}$. Therefore, we have

$$
G-v ¥ D_{n-1, k}, C_{8} \uplus \frac{n-9}{2} K_{2}, C_{10} \uplus \frac{n-11}{2} K_{2} \text { and }\left(C_{8} * K_{2}\right) \uplus \frac{n-11}{2} K_{2} .
$$

By induction hypothesis, we have $\operatorname{mi}(G-v) \leqslant r^{n-3}$. Thus,

$$
\begin{aligned}
\operatorname{mi}(G) & \leqslant \operatorname{mi}(G-v)+\operatorname{mi}\left(G-N_{G}[v]\right) & \quad \text { (by Lemma 1) } \\
& \leqslant r^{n-3}+r^{n-5} & \text { (by Lemma 6) } \\
& =3 r^{n-5} . &
\end{aligned}
$$

The equality in (14) holds if and only if $G-v \cong H_{0}(6) \uplus \frac{n-7}{2} K_{2}, H_{0}(8) \uplus \frac{n-9}{2} K_{2}, H_{0}(10) \uplus \frac{n-11}{2} K_{2}$, $H_{1}(k) \uplus \frac{n-k-1}{2} K_{2}, H_{2}(k) \uplus \frac{n-k-1}{2} K_{2}$, or $H_{3}(k) \uplus \frac{n-k-1}{2} K_{2}$ and $G-N_{G}[v] \cong F_{1}(n-5)$. Since $G$ is connected and $G-N_{G}[v]$ has no isolated vertex, $G-v$ contains no $K_{2}$ as a component, i.e., $G-v \cong H_{0}(6), H_{0}(8), H_{0}(10), H_{1}(n-1), H_{2}(n-1)$, or $H_{3}(n-1)$. However, $G-N_{G}[v]$ is obtained from $G-v$ by deleting four independent vertices which is impossible. Hence $\operatorname{mi}(G)<3 r^{n-5}$.

Case 2. Every vertex of degree $\geqslant 4$ is in all cycles of $G$.

First we consider $\delta(G)=1$. Choose an edge $u v \in E_{G}$ with $d(v)=1$. Let $G_{1}=G-\{u, v\}$ and $G_{2}=G-N[u]$. We distinguish the following two possible subcases to show our results.

Subcase 2.1. $n$ is odd.

If $d(u)=2$, then $G_{1}$ is a connected graph with cycles and $G_{2} \not \frac{n-3}{2} K_{2}$. By induction hypothesis and Lemma 5 and Theorem 1, we have $\operatorname{mi}\left(G_{1}\right) \leqslant 3 r^{n-7}$ and $\operatorname{mi}\left(G_{2}\right) \leqslant \max \left\{3 r^{n-7}, r^{n-5}+r^{n-9}\right\}=3 r^{n-7}$. By Lemma 1, we get

$$
\operatorname{mi}(G)=\operatorname{mi}\left(G_{1}\right)+\operatorname{mi}\left(G_{2}\right) \leqslant 3 r^{n-7}+3 r^{n-7}=3 r^{n-5},
$$

the equality holds if and only if $G_{1} \cong O_{1}(n-2)$ or $C_{6} * K_{1}, G_{2} \cong P_{4} \uplus \frac{n-7}{2} K_{2}$, i.e., $G \cong O_{1}(n)$.

If $d(u) \geqslant 3$, by Lemmas 1 and 6 , we obtain that

$$
\operatorname{mi}(G)=\operatorname{mi}\left(G_{1}\right)+\operatorname{mi}\left(G_{2}\right) \leqslant r^{n-2-1}+r^{n-4-1}=3 r^{n-5},
$$


the equality holds if and only if $G_{1} \cong F_{1}(n-2), G_{2} \cong F_{1}(n-4)$ or $F_{1}(n-5)$. If $G_{2} \cong F_{1}(n-4)$, we have $d(u)=3$. In this situation, we get $G \cong C_{6} * K_{1}$. If $G_{2} \cong F_{1}(n-5)$, we have $d(u)=4 . G_{2}$ is obtained from $G_{1}$ by deleting three independent vertices which is impossible.

Subcase 2.2. $n$ is even.

- $d(u)=2$. In this subcase, we know that $G_{1}$ is a connected graph with cycles. Note that $G \nsucceq C_{8} * K_{2}$, hence $G_{1} \nsucceq C_{8}$. By Theorem 1 , we have $\operatorname{mi}\left(G_{1}\right) \leqslant r^{n-4}+1$. On the other hand, notice that $G \supsetneqq D_{n, k}$, hence if $G_{2} \cong B_{n-3, k}$, we obtain that $G \cong H_{1}(n), H_{2}(n)$, or $H_{3}(n)$, our result holds. If $G_{2} \nsucceq B_{n-3, k}$, by induction hypothesis or Lemma 7 , we have $\operatorname{mi}\left(G_{2}\right) \leqslant \max \left\{r^{n-4}-1,3 r^{n-8}\right\}=r^{n-4}-1$. Combining with Lemma 1, we have

$$
\operatorname{mi}(G)=\operatorname{mi}\left(G_{1}\right)+\operatorname{mi}\left(G_{2}\right) \leqslant r^{n-4}+1+r^{n-4}-1=r^{n-2},
$$

the equality holds if and only if $G_{1} \cong B_{n-2}, C_{10}$ or $C_{8} * K_{2}$ and $\operatorname{mi}\left(G_{2}\right)=r^{n-4}-1$, but there is no such bipartite graph.

- $d(u)=3$. If $G_{1}$ is disconnected, then $G_{1}$ must have cycles and $G_{2} \not \frac{n-4}{2} K_{2}$ since $G$ have cycles. Thus, by Lemma 5 and Theorem 1 , we get $\operatorname{mi}\left(G_{1}\right) \leqslant r^{n-4}+r^{n-8}=5 r^{n-8}$ and $\operatorname{mi}\left(G_{2}\right) \leqslant \max \left\{3 r^{n-8}, r^{n-6}+\right.$ $\left.r^{n-10}\right\}=3 r^{n-8}$. By Lemma 1 , we have

$$
\operatorname{mi}(G)=\operatorname{mi}\left(G_{1}\right)+\operatorname{mi}\left(G_{2}\right) \leqslant 5 r^{n-8}+3 r^{n-8}=r^{n-2},
$$

the equality holds if and only if $G_{1} \cong C_{6} \uplus \frac{n-8}{2} K_{2}$ and $G_{2} \cong P_{4} \uplus \frac{n-8}{2} K_{2}$, but there is no such bipartite graph. If $G_{1}$ is connected and $G_{2} \cong \frac{n-4}{2} K_{2}$, then we have $G \cong H_{2}(n)$ and $\operatorname{mi}(G)=r^{n-2}$, the result holds. If $G_{1}$ is connected and $G_{2} \nsucceq \frac{n-4}{2} K_{2}$, then for $n=6$, we get $G_{2}=2 K_{1}$ and $G \cong H_{0}(6)$, the result holds. We assume $n \geqslant 8$. Obviously, $G_{1} \nsucceq C_{8}$ since $G \varsubsetneqq C_{8} * K_{2}$. By Lemmas 3,5 and Theorem 1 , we have $\operatorname{mi}\left(G_{1}\right) \leqslant r^{n-4}+1, \operatorname{mi}\left(G_{2}\right) \leqslant \max \left\{3 r^{n-8}, r^{n-6}+r^{n-10}\right\}=3 r^{n-8}$. Thus,

$$
\begin{aligned}
\operatorname{mi}(G) & \left.=\operatorname{mi}\left(G_{1}\right)+\operatorname{mi}\left(G_{2}\right) \quad \text { (by Lemma } 1\right) \\
& \leqslant r^{n-4}+1+3 r^{n-8} \\
& =7 r^{n-8}+1 \\
& \leqslant r^{n-2}
\end{aligned}
$$

The equality in (15) holds if and only if $G_{1} \cong T_{1}(n-2)$, or $B_{n-2}, G_{2} \cong P_{4} \uplus \frac{n-8}{2} K_{2}$; while the equality in (16) holds if and only if $n=8$, i.e., $G_{1} \cong P_{4} * K_{2}, C_{6}$ or $P_{6}$ and $G_{2} \cong P_{4}$, but there is no such bipartite graph.

- $d(u) \geqslant 4$. Note that $G$ contains cycles, it is easy to see that $G_{1} \not \frac{n-2}{2} K_{2}$. By Lemmas 5,6 and Theorem 1, we get $\operatorname{mi}\left(G_{1}\right) \leqslant \max \left\{3 r^{n-6}, r^{n-4}+r^{n-8}\right\}=3 r^{n-6}$ and $\operatorname{mi}\left(G_{2}\right) \leqslant r^{n-6}$. By Lemma 1 ,

$$
\operatorname{mi}(G)=\operatorname{mi}\left(G_{1}\right)+\operatorname{mi}\left(G_{2}\right) \leqslant 3 r^{n-6}+r^{n-5-1}=r^{n-2},
$$

the equality holds if and only if $G_{1} \cong P_{4} \uplus \frac{n-6}{2} K_{2}, G_{2} \cong F_{1}(n-5)$ or $F_{1}(n-6)$. Note that $G_{2}$ is obtained from $G_{1}$ by deleting three or four independent vertices, hence there is no such bipartite graph of order $n$. Hence $\operatorname{mi}(G)<r^{n-2}$.

Now we consider $\delta(G) \geqslant 2$. In this subcase, we used the following two facts (for their proofs one may be referred to the Appendix). 
Fact 1 Suppose $n \geqslant 7$ is odd and each vertex of degree $\geqslant 4$ is in all cycles of $G$, then $\operatorname{mi}(G)<3 r^{n-5}$.

Fact 2 Suppose $n \geqslant 6$ is even and each vertex of degree $\geqslant 4$ is in all cycles of $G$, then $\operatorname{mi}(G) \leqslant r^{n-2}$. The equality holds if and only if $G \cong H_{1}(n)$.

Obviously, in this case, if $\delta(G) \geqslant 2$, Theorem 2 holds directly from Facts 1 and 2.

\section{Concluding remark}

In view of Theorems 1 and 2(i), the disconnected graphs among $\mathscr{B}_{n}$ with the first-, second-, $\ldots, \frac{n-2}{2}$-th largest number of maximal independent sets are characterized, while the connected graphs in $\mathscr{B}_{n}$ having the largest and the second largest number of maximal independent sets are determined; whereas in view of Theorem 2(ii), graphs among $\mathscr{B}_{n}^{\prime}$ having the largest number of maximal independent sets are identified.

\section{Acknowledgements}

The authors would like to express their sincere gratitude to the referees for a very careful reading of the paper and for all of their insightful comments and valuable suggestions, which led to a number of improvements in this paper. This work was supported by the National Natural Science Foundation of China (Grant No. 11271149) and the Special Fund for Basic Scientific Research of Central Colleges (Grant No. CCNU13F020).

\section{References}

[1] S. Arumugam, T.W. Haynes, M.A. Henning, Y. Nigussie, Maximal independent sets in minimum colorings, Discrete Math. 311 (13) (2011) 1158-1163.

[2] S. Basagni, A distributed algorithm for finding a maximal weighted independent set in wireless network. In: Proceedings of the 11th IASTED international conference on parallel and distributed computing and systems(PDCS), 1999, pp 517-522.

[3] B.A. Bondy, U.S.R. Murty, Graph Theory with Applications, Macmillan, London, 1976.

[4] D. Duffus, P. Frankl, V. Rödl, Maximal independent sets in bipartite graphs obtained from Boolean lattices, European J. Combin. 32 (1) (2011) 1-9.

[5] S. El-Basil, Binomial-combinatorial properties of Clar structures, Discrete Appl. Math. 19(1-3)(1998) 145-156.

[6] H.B. Hua, Y.P. Hou, On graphs with the third largest number of maximal independent sets, Inform. Process. Lett. 109 (4) (2009) 248-253.

[7] M. Hujter, Z. Tuza, The number of maximal independent sets in triangle-free graphs, SIAM J. Discrete Math. 6 (1993) 284-288.

[8] Z.M. Jin, X.L. Li, Graphs with the second largest number of maximal independent sets, Discrete Math. 308 (2008) 5864-5870.

[9] Z.M. Jin, S.H.F. Yan, Trees with the second and third largest number of maximal independent sets, Ars Combin. 93 (2009) 341-351.

[10] M.J. Jou, The number of maximal independent sets in graphs, Master Thesis, Department of Mathematics, National Central University, Taiwan, 1991.

[11] M.J. Jou, G. J. Chang, Servey on counting maximal independent sets, in: S.Tangmance, E.Schulz(Eds.), Proceedings of the second Asian Mathematics Conference, World Scientific, Singapore, 1995, pp. 265-275. 
[12] M.J. Jou, G. J. Chang, Algorithmic aspects of counting independent sets, Ars Combin. 65 (2002) 265-277.

[13] M.J. Jou, G. J. Chang, Maximal independent sets in graphs with at most one cycle, Discrete Appl. Math. 79 (1997) 67-73 .

[14] M.J. Jou, J.J. Lin, Trees with the second largest number of maximal independent sets, Discrete Math. 309 (2009) 4469-4474.

[15] M.J. Jou, The second largest number of maximal independent sets in connected graphs with at most one cycle, J. Comb. Optim. 24 (2012) 192-201.

[16] S.C. Li, W. Jing, Maximal independent sets in graphs with cyclomatic number at most two. Util. Math. 83 (2010) 107-120.

[17] J.Q. Liu, Maximal independent sets in bipartite graphs, J. Graph Theory, 17 (1993) 495-507.

[18] J.W. Moon, L. Moser, On Cliques in graphs, Israel J. Math. 3 (1965) 23-28.

[19] T. Moscibroda, R. Wattenhofer, Efficient computation of maximal independent sets in unstructured multi-hop radio network. In: Proc of 1-st international conference on mobile ad hoc and sensor systems, 2004, pp 51-59.

[20] C. Ortiz, M. Villanueva, Maximal independent sets in caterpillar graphs, Discrete Appl. Math. 160 (3) (2012) 259-266.

[21] N. Rhodes, P. Willett, A. Calvet, J.B. Dunbar, C. Humblet, CLIP: similarity searching of 3D databases using clique detection, J. Chem. Inf. Comput. Sci. 43 (2) (2003) 443-448.

[22] G.C. Ying, K.K. Meng, B.E. Sagan, V.R. Vatter, Maximal independent sets in graphs with at most $r$ cycles, J. Graph Theory 53 (4) (2006) 270-282.

\section{Appendix}

In the appendix, we present the proofs for Facts 1 and 2.

Proof of Fact 1: We first assume that $G$ has a vertex $v$ of degree 3 such that $G-v$ has cycles. Note that $G$ contains no odd cycles and $\delta(G) \geqslant 2$, hence $G-v$ (resp. $G-N_{G}[v]$ ) does not contain $K_{2}$ as a component.

- $G-v \not D_{n-1, k}$. Then we are to show that $G-v \nsucceq C_{8} \uplus \frac{n-9}{2} K_{2}, C_{10} \uplus \frac{n-11}{2} K_{2}$ and $\left(C_{8} * K_{2}\right) \uplus \frac{n-11}{2} K_{2}$ by contradiction.

If $G-v \cong C_{8} \uplus \frac{n-9}{2} K_{2}$, i.e., $G-v \cong C_{8}$. Notice that $d(v)=3$, we have $G-N_{G}[v] \cong 2 K_{1} \uplus P_{3}$. So we obtain that the graph $G$ is depicted in Fig. 3(a). By Lemma 1, we get $\operatorname{mi}(G) \leqslant \operatorname{mi}(G-u)+\operatorname{mi}(G-N[u])=7+4=11<3 r^{4}=12$.

If $G-v \cong C_{10} \uplus \frac{n-11}{2} K_{2}$, i.e., $G-v \cong C_{10}$. Note that $d(v)=3$, we have $G-N_{G}[v] \cong 2 K_{1} \uplus P_{5}$ or $K_{1} \uplus 2 P_{3}$. By Lemma 1, we get $\operatorname{mi}(G) \leqslant \operatorname{mi}(G-v)+\operatorname{mi}\left(G-N_{G}[v]\right)=17+4=21<3 r^{6}=24$.

If $G-v \cong\left(C_{8} * K_{2}\right) \uplus \frac{n-11}{2} K_{2}$, i.e., $G-v \cong C_{8} * K_{2}$. Note that $d(v)=3$, we have $G-N_{G}[v] \cong 2 K_{1} \uplus P_{5}, K_{1} \uplus 2 P_{3}$, $K_{1} \uplus\left(K_{1} *\left(K_{2} \uplus P_{3}\right)\right)$ or $P_{3} \uplus K_{1,3}$. By Lemma 1, we get $\operatorname{mi}(G) \leqslant \operatorname{mi}(G-v)+\operatorname{mi}\left(G-N_{G}[v]\right)=17+4=21<3 r^{6}=24$. Hence, we have

$$
G-v \varsubsetneqq D_{n-1, k}, C_{8} \uplus \frac{n-9}{2} K_{2}, C_{10} \uplus \frac{n-11}{2} K_{2} \text { and }\left(C_{8} * K_{2}\right) \uplus \frac{n-11}{2} K_{2} .
$$

By induction hypothesis, $\operatorname{mi}(G-v) \leqslant r^{n-3}$. In view of Lemmas 1 and 6 , we have

$$
\begin{aligned}
\operatorname{mi}(G) & \leqslant \operatorname{mi}(G-v)+\operatorname{mi}\left(G-N_{G}[v]\right) \\
& \leqslant r^{n-3}+r^{n-4-1} \\
& =3 r^{n-5} .
\end{aligned}
$$

The equality in (A.1) holds if and only if $G-v \cong H_{0}(n-1), H_{1}(n-1), H_{2}(n-1)$, or $H_{3}(n-1)$ for $n=7,9,11$ and $G-v \cong H_{1}(n-1), H_{2}(n-1)$, or $H_{3}(n-1)$ for $n \geqslant 13, G-N_{G}[v] \cong F_{1}(n-4)$, i.e., $G-N_{G}[v] \cong T_{1}(n-4)$. Note that $G-N_{G}[v]$ is obtained from $G-v$ by deleting three independent disjoint vertices, but there is no such bipartite graph of order $n$. Hence $\operatorname{mi}(G)<3 r^{n-5}$. 
- $G-v \cong D_{n-1, k}$, i.e., $G-v \cong B_{n-1}$. In this case, we have $\operatorname{mi}(G-v)=r^{n-3}+1$. Furthermore, $G-N_{G}[v]$ is either an acyclic graph which is not isomorphic to $B_{n-4, k}$ or a bipartite graph containing cycles. It follows from induction hypothesis or Lemma 7 that $\operatorname{mi}\left(G-N_{G}[v]\right) \leqslant \max \left\{r^{n-4-1}-1,3 r^{n-4-5}\right\}=r^{n-5}-1$. Together with Lemma 1, we have

$$
\begin{aligned}
\operatorname{mi}(G) & \leqslant \operatorname{mi}(G-v)+\operatorname{mi}\left(G-N_{G}[v]\right) \\
& \leqslant r^{n-3}+1+r^{n-5}-1 \\
& =3 r^{n-5} .
\end{aligned}
$$

The equality in (A.2) holds if and only if $G-v \cong B_{n-1}, \operatorname{mi}\left(G-N_{G}[v]\right)=r^{n-5}-1$. Note that $G$ is a bipartite graph with $\delta(G) \geqslant 2$, hence $G-v$ must be the graph as depicted in Fig. 3(b). $G-N_{G}[v]$ is obtained from $G-v$ by deleting three independent vertices. Elementary calculation yields $\operatorname{mi}\left(G-N_{G}[v]\right)<r^{n-5}-1$. Hence, $\operatorname{mi}(G)<3 r^{n-5}$.

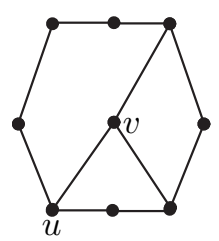

(a)

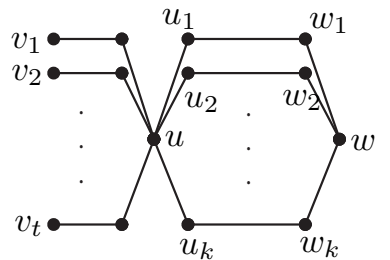

(b)

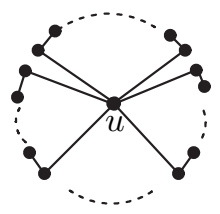

$(c)$

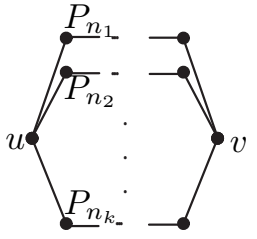

(d)

Fig. 3: Graphs used in the proof of Fact 1.

Now, we consider the case that each vertex of degree $\geqslant 3$ is in all cycles of $G$. Then $G$ must be a graph with the structures as depicted in Figs. 3(c) or 3(d). Assume that the vertex $u$ is in all cycles of $G$, then $d(u) \geqslant 3$. Note that $n$ is odd and $\delta(G) \geqslant 2$, hence $G-u \nsubseteq B_{n-1, k}$ and $G-u$ contains no cycles. By Lemma 7, we get $\operatorname{mi}(G-u) \leqslant r^{n-3}$. Thus, we have

$$
\begin{array}{rlr}
\operatorname{mi}(G) & \leqslant \operatorname{mi}(G-u)+\operatorname{mi}(G-N[u]) & \quad \text { (by Lemma } 1) \\
& \leqslant r^{n-3}+r^{n-4-1} \quad(\text { by Lemma } 6) \\
& =3 r^{n-5} . &
\end{array}
$$

The equality in (A.3) holds if and only if $\operatorname{mi}(G-u)=r^{n-3}, G-N[u] \cong F_{1}(n-4)$ or $F_{1}(n-5)$, i.e., $G-N[u] \cong T_{1}(n-4)$ or $T_{1}(n-5)$. Notice that $\delta(G) \geqslant 2$ and $u$ is in all cycles of $G$, it is straightforward to check that $n \in\{7,9,11\}$ and $G-N[u] \not$ $T_{1}(n-5)$. So we get that $G$ is a graph of the structure in Fig. 3(d). That is to say, $G$ has exactly two vertices of degree $\geqslant 3$, say $u$ and $v$. It follows that $d(u)=d(v)=d$ and $G-\{u, v\}=P_{n_{1}} \uplus \cdots \uplus P_{n_{k}}$ with $n_{1} \geqslant n_{2} \geqslant \cdots \geqslant n_{k}$. If $n=7$, then $n_{1}=3, n_{2}=1, n_{3}=1$. By elementary calculation, $\operatorname{mi}(G)=5<6$. If $n=9$, then $n_{1}=5, n_{2}=1, n_{3}=1$. By Lemma $1, \operatorname{mi}(G) \leqslant \operatorname{mi}(G-u)+\operatorname{mi}(G-N[u])=4+7=11<12$. If $n=11$, then $n_{1}=n_{2}=n_{3}=3$. By Lemma 1 , $\operatorname{mi}(G) \leqslant \operatorname{mi}(G-u)+\operatorname{mi}(G-N[u])=13+8=21<24$. Hence, we obtain $\operatorname{mi}(G)<3 r^{n-5}$. Thus, Fact 1 holds.

Further on we need the following lemma to prove Fact 2.

Lemma A Suppose $n \geqslant 6$ is even. If $G$ has a path $P_{5}=u_{1} u_{2} u_{3} u_{4} u_{5}$ such that $d\left(u_{3}\right)=2, G-\left\{u_{1}, u_{2}, u_{3}, u_{4}, u_{5}\right\}$ has cycles and $G-\left\{u_{2}, u_{3}, u_{4}, u_{5}\right\} \nsubseteq D_{n-4, k}, C_{8} \uplus \frac{n-12}{2} K_{2}, C_{10} \uplus \frac{n-14}{2} K_{2}$ and $\left(C_{8} * K_{2}\right) \uplus \frac{n-14}{2} K_{2}$, then $\operatorname{mi}(G)<r^{n-2}$.

Proof: From the assumption it follows that $G_{1}:=G-\left\{u_{1}, u_{2}, u_{3}\right\}, G_{2}:=G-\left\{u_{2}, u_{3}, u_{4}\right\}$ and $G_{3}:=G-\left\{u_{2}, u_{3}, u_{4}, u_{5}\right\}$, respectively, has cycles and $G_{3} \nsucceq D_{n-4, k}, C_{8} \uplus \frac{n-12}{2} K_{2}, C_{10} \uplus \frac{n-14}{2} K_{2}$ and $\left(C_{8} * K_{2}\right) \uplus \frac{n-14}{2} K_{2}$. Hence, by Lemma 1 we obtain

$$
\begin{array}{rlr}
\operatorname{mi}(G) & \leqslant \operatorname{mi}\left(G-u_{2}-u_{4}\right)+\operatorname{mi}\left(G-u_{2}-u_{4}-N\left(u_{4}\right)\right)+\operatorname{mi}\left(G-N\left[u_{2}\right]\right) \\
& =\operatorname{mi}\left(G_{2}\right)+\operatorname{mi}\left(G_{3}\right)+\operatorname{mi}\left(G_{1}\right) & \\
& \leqslant 3 r^{n-3-5}+r^{n-4-2}+3 r^{n-3-5} & \text { (by induction hypothesis) } \\
& =r^{n-2} . &
\end{array}
$$


The equality in (A.4) holds if and only if $d\left(u_{2}\right)=d\left(u_{4}\right)=2, G_{1}=G_{2} \cong\left(C_{6} * K_{1}\right) \uplus \frac{n-10}{2} K_{2}$ or $O_{1}(k) \uplus \frac{n-k-3}{2} K_{2}$, and $G_{3} \cong H_{0}(6) \uplus \frac{n-10}{2} K_{2}, H_{0}(8) \uplus \frac{n-12}{2} K_{2}, H_{0}(10) \uplus \frac{n-14}{2} K_{2}, H_{1}(k) \uplus \frac{n-k-4}{2} K_{2}, H_{2}(k) \uplus \frac{n-k-4}{2} K_{2}$, or $H_{3}(k) \uplus$ $\frac{n-k-4}{2} K_{2}$. Since $\delta(G) \geqslant 2, G_{1}, G_{2}$ contains no $K_{2}$ as a component, i.e., $G_{1}=G_{2} \cong C_{6} * K_{1}$ or $O_{1}(n-3)$. If $G_{1}=G_{2} \cong$ $C_{6} * K_{1}$, we get $G \cong H_{0}(10)$ or the graph depicted in Fig. 4(c). But this implies $G-\left\{u_{1}, u_{2}, u_{3}, u_{4}, u_{5}\right\}$ has no cycles, a contradiction. If $G_{1}=G_{2} \cong O_{1}(n-3)$, we get two such graphs which contain odd cycles, a contradiction. That is to say, the equality in (A.4) does not hold. Hence, $\operatorname{mi}(G)<r^{n-2}$, as desired.

Proof of Fact 2: We say a vertex $v$ is good if $d(v)=3$ and $G-v$ has cycles. Since $\delta(G) \geqslant 2$ and $G$ is bipartite, then $G-N_{G}[v] \nRightarrow B_{n-4, k}$ for any good vertex $v$.

First we consider that $G$ has good vertices by distinguishing the following two possible cases.

Case 1. For all good vertices $v, G-N_{G}[v] \cong D_{n-4, k}$.

For convenience, let $G_{1}=G-N_{G}[v]$. Note that $\delta(G) \geqslant 2$ and $G$ is bipartite, hence $G_{1}$ must be connected and $G_{1} \cong B_{n-4}$. Furthermore, $G_{1}$ must be a graph with the structure shown in Fig. 3(b). Clearly, if $t \geqslant 1$, then $N(w) \cap N(v)=\emptyset$; if $t=0$, then either $N(w) \cap N(v)=\emptyset$ or $N(u) \cap N(v)=\emptyset$. Hence, without loss of generality, assume $N(w) \cap N(v)=\emptyset$. Note that $d(v)=3$ and $\delta(G) \geqslant 2$, hence $G-w$ has cycles. Thus, by the assumption that each vertex of degree $\geqslant 4$ is in all cycles of $G$, it follows that $2 \leqslant d(x) \leqslant 3$ for $x=w$ or $x \in N(v)$.

If $d(u) \leqslant 3$, note that $\delta(G) \geqslant 2$ and $G-N_{G}[v] \cong D_{n-4, k}$ for every good vertex $v, G$ must be the graph shown in Figs. 4(a) or 4(b). For Fig. 4(a), $\operatorname{mi}(G) \leqslant \operatorname{mi}(G-v)+\operatorname{mi}\left(G-N_{G}[v]\right)=8+5=13<16$; For Fig. $4(\mathrm{~b})$, $\operatorname{mi}(G) \leqslant$ $\operatorname{mi}(G-v)+\operatorname{mi}\left(G-N_{G}[v]\right)=6+5=11<16$, they are not the extremal graphs.

If $d(u) \geqslant 4$, then $G$ has at most one edge between $N(v)$ and $A=\left\{u_{j}: 1 \leqslant j \leqslant k\right\} \cup\left\{w_{j}: 1 \leqslant j \leqslant k\right\}$. Consequently, we have $d(w)=2$. Otherwise, $w$ is a good vertex of $G$, so in $G-N[w]$, there is at least one vertex $y \in N(u)$ such that $d(y)=1$. Obviously, $G-N[w] \varsubsetneqq D_{n-4, k}$, a contradiction. Similarly, we can conclude that each vertex in $N(v)$ has degree 2. Now, let $P_{5}=u_{1} w_{1} w w_{2} u_{2}$. Obviously, $d(w)=2, G-\left\{u_{1}, w_{1}, w, w_{2}, u_{2}\right\}$ has cycles and $G-\left\{w_{1}, w, w_{2}, u_{2}\right\} \not$ $D_{n-4, k}, C_{8} \uplus \frac{n-12}{2} K_{2}, C_{10} \uplus \frac{n-14}{2} K_{2}$ and $\left(C_{8} * K_{2}\right) \uplus \frac{n-14}{2} K_{2}$. By Lemma A, we obtain that $\operatorname{mi}(G)<r^{n-2}$.

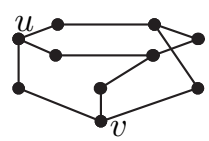

(a)

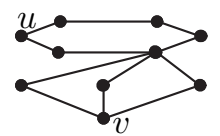

(b)

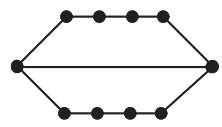

(c)

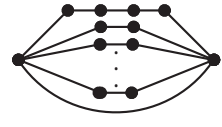

(d)

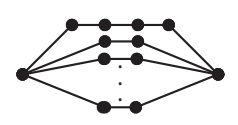

(f)

Fig. 4: Graphs used in the proof of Fact 2.

Case 2. There exists a good vertex $v$ such that $G-N_{G}[v] \nsupseteq D_{n-4, k}$.

In this case, we are to show that $G_{1}:=G-N_{G}[v] \supsetneqq C_{8} \uplus \frac{n-9}{2} K_{2}, C_{10} \uplus \frac{n-11}{2} K_{2}$ and $\left(C_{8} * K_{2}\right) \uplus \frac{n-11}{2} K_{2}$. In fact, if $G_{1} \cong C_{8} \uplus \frac{n-9}{2} K_{2}$, then there exist eight such graphs, i.e., $G^{1}, G^{2}, \ldots, G^{8}$; if $G_{1} \cong C_{10} \uplus \frac{n-11}{2} K_{2}$, then there exist thirteen such graphs, i.e., $G^{9}, G^{10}, \ldots, G^{21}$; if $G_{1} \cong\left(C_{8} * K_{2}\right) \uplus \frac{n-11}{2} K_{2}$, then there exist twenty-five such graphs, i.e., $G^{22}, G^{23}, \ldots, G^{46}$, where $G^{1}, G^{2}, \ldots, G^{46}$ are depicted in Fig. 5. By direct computation, we obtain $\operatorname{mi}(G)<r^{n-2}$. Hence, $G_{1} \nsucceq C_{8} \uplus \frac{n-9}{2} K_{2}, C_{10} \uplus \frac{n-11}{2} K_{2}$ and $\left(C_{8} * K_{2}\right) \uplus \frac{n-11}{2} K_{2}$. By Lemma 7 (if $G_{1}$ is acyclic) or by induction hypothesis (if $G_{1}$ contains cycles), we obtain that $\operatorname{mi}\left(G_{1}\right) \leqslant r^{n-6}$. Thus, we obtain that

$$
\begin{aligned}
\operatorname{mi}(G) & \leqslant \operatorname{mi}(G-v)+\operatorname{mi}\left(G_{1}\right) & \text { (by Lemma 1) } \\
& \leqslant 3 r^{n-6}+r^{n-6} & \text { (by induction hypothesis) } \\
& =r^{n-2} . &
\end{aligned}
$$

The equality in (A.5) holds if and only if $G-v \cong\left(C_{6} * K_{1}\right) \uplus \frac{n-8}{2} K_{2}$ or $O_{1}(k) \uplus \frac{n-1-k}{2} K_{2}, \operatorname{mi}\left(G_{1}\right)=r^{n-6}$. Note that $\delta(G) \geqslant 2$ and $G$ contains no odd cycles, $G-v$ contains no $K_{2}$ as a component, hence $G-v \cong C_{6} * K_{1}$ or $O_{1}(n-1)$. If $G-v \cong C_{6} * K_{1}$, there are two such graphs. By a straightforward calculation, $\operatorname{mi}(G)=6$ or $7<r^{6}=8$. If $G-v \cong O_{1}(n-1)$, then $6 \leqslant n \leqslant 10$ since $d(v)=3$ and $\delta(G) \geqslant 2$. For $n=6$, there is one such graph. By a straightforward calculation, $\operatorname{mi}(G)=3<4$. For $n=8$, there are two such graphs. By a straightforward calculation, $\operatorname{mi}(G)=7<8$. For $n=10$, we get 


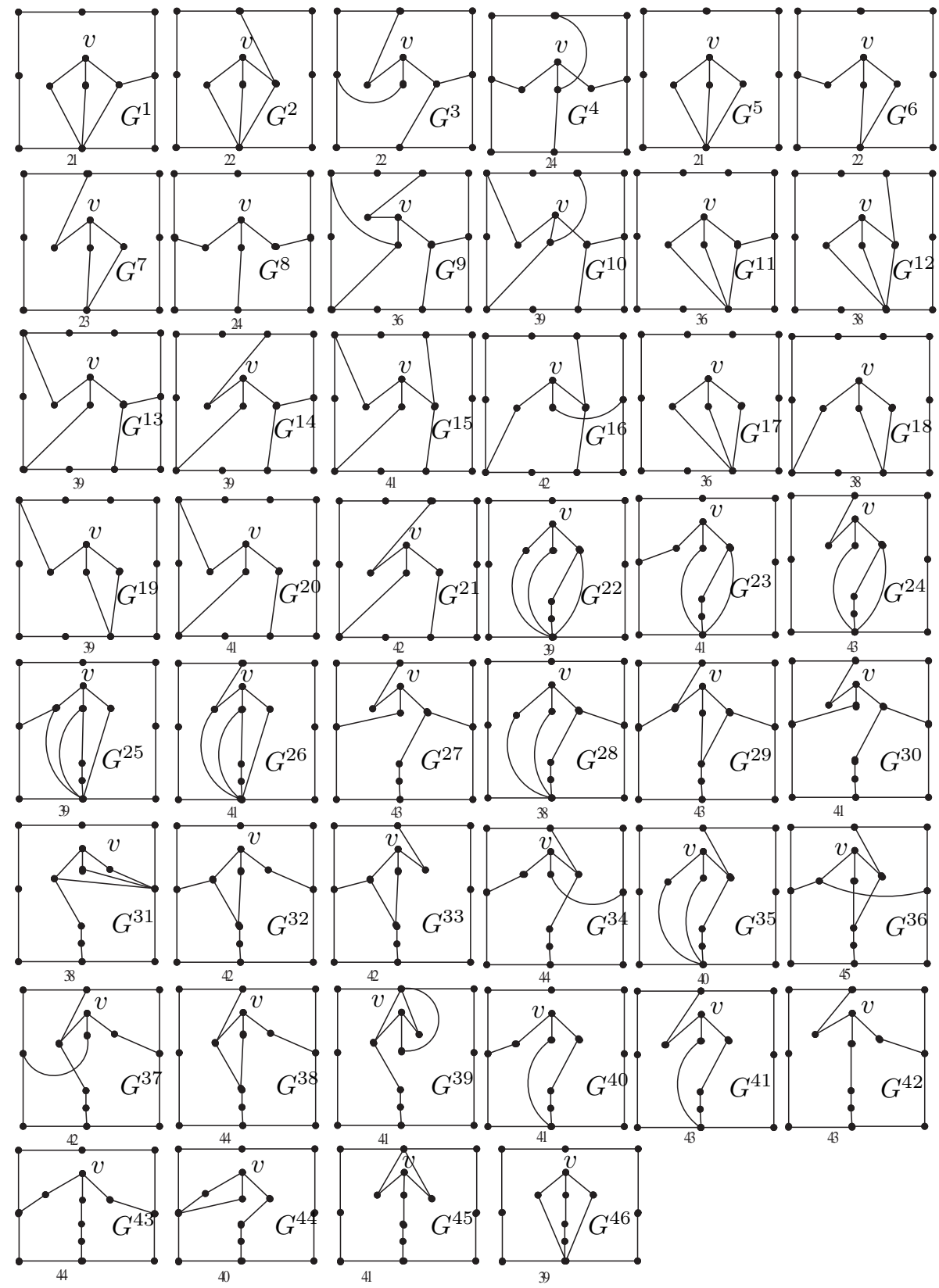

Fig. 5: Graphs $G^{1}, G^{2}, \ldots, G^{46}$ used in Case 2 of Fact 2, in which each number below the graph $G^{i}$ is an upper bound of $\operatorname{mi}\left(G^{i}\right), i=1,2, \ldots, 46$. 
$G_{1} \cong C_{4} * 2 K_{1}$. By Lemma $1, \operatorname{mi}(G) \leqslant \operatorname{mi}(G-v)+\operatorname{mi}\left(G_{1}\right)=12+3=15<16$. Hence, $\operatorname{mi}(G)<r^{n-2}$. The result holds.

Now we consider that $G$ has no good vertex, that is to say, each vertex of degree $\geqslant 3$ must be contained in all cycles of $G$. This implies that $G$ must be the graph with structures shown in Figs. 3(c) or 3(d). By Lemma 8 and $G \neq C_{6}, C_{8}$ and $C_{10}$, we assume that $G$ is not a cycle. If $G$ is a graph of the structure in Fig. 3(c), then $G$ has only one vertex $u$ of degree $\geqslant 3$. Since $G$ is a bipartite graph of even order, then $d(u) \geqslant 6$ and $G-u$ consists of $k \geqslant 3$ disjoint paths of even length, say $P_{n_{1}}, P_{n_{2}}, \ldots, P_{n_{k}}$ (see Fig. 3(c)), where $k=\frac{d(u)}{2}$. By Lemmas 2 and 3, it follows that

$$
\operatorname{mi}(G-u)=\prod_{j=1}^{k} \operatorname{mi}\left(P_{n_{j}}\right) \leqslant \prod_{j=1}^{k} r^{n_{j}-1} \leqslant r^{n-4}
$$

and so

$$
\begin{array}{rlr}
\operatorname{mi}(G) & \leqslant \operatorname{mi}(G-u)+\operatorname{mi}\left(G-N_{G}[u]\right) & \text { (by Lemma 1) } \\
& \leqslant r^{n-4}+r^{n-8} & \text { (by Lemma 6) } \\
& =5 r^{n-8}<r^{n-2} &
\end{array}
$$

The result holds.

Suppose $G$ is a graph of the structure in Fig. 3(d), then $G$ has exactly two vertices degree $\geqslant 3$, say $u, v$. It follows that $d(u)=d(v)=d \geqslant 3$ and $G-\{u, v\}$ consists of $k$ disjoint paths, say $P_{n_{1}}, P_{n_{2}}, \ldots, P_{n_{k}}$ with $n_{1} \geqslant n_{2} \geqslant \cdots \geqslant n_{k}$, where $k=d$ if $u$ and $v$ are not adjacent and $k=d-1$ otherwise. Since $G$ is a bipartite graph, either all $n_{j}$ 's are odd or all $n_{j}$ 's are even.

If all $n_{j}$ 's are odd, we have $k=d \geqslant 4$. By Lemmas 2 and 3 , we get

$$
\operatorname{mi}(G-u-v)=\prod_{j=1}^{k} \operatorname{mi}\left(P_{n_{j}}\right) \leqslant \prod_{j=1}^{k} r^{n_{j}-1} \leqslant r^{n-6} .
$$

This gives

$$
\begin{array}{rlr}
\operatorname{mi}(G) & \leqslant \operatorname{mi}(G-u-v)+\operatorname{mi}\left(G-u-v-N_{G}(v)\right)+\operatorname{mi}\left(G-N_{G}[u]\right) \text { (by applying Lemma } 1 \text { twice) } \\
& \leqslant r^{n-6}+r^{n-6}+r^{n-5-1} & \text { (by Lemma 6) } \\
& =3 r^{n-6}<r^{n-2} . &
\end{array}
$$

Now we consider that all $n_{j}$ 's are even. Let $P_{n_{1}}=u_{1} u_{2} \ldots u_{n_{1}}$. By Lemma A, we assume $n_{1} \leqslant 4$. If $n_{1}=4$, then both $L_{1}=G-\left\{u_{1}, u_{2}, u_{3}\right\}$ and $L_{2}=G-\left\{u_{2}, u_{3}, u_{4}\right\}$ have cycles and $L_{3}=G-\left\{u_{1}, u_{2}, u_{3}, u_{4}, v_{1}\right\}$ is a tree. Furthermore, $L_{3} \not T_{1}(n-4)$ unless $G$ is one of the graphs shown in Figures 4(c), 4(d) and 4(f). If $L_{3} \not T_{1}(n-4)$, by Lemma 1 we get

$$
\begin{aligned}
\operatorname{mi}(G) & \leqslant \operatorname{mi}\left(G-u_{1}-u_{3}\right)+\operatorname{mi}\left(G-u_{1}-u_{3}-N\left(u_{1}\right)\right)+\operatorname{mi}\left(G-N\left[u_{3}\right]\right) \\
& =\operatorname{mi}\left(L_{1}\right)+\operatorname{mi}\left(L_{3}\right)+\operatorname{mi}\left(L_{2}\right) \\
& \leqslant 3 r^{n-8}+r^{n-6}+3 r^{n-8} \quad \text { (by induction hypothesis and Lemma 6) } \\
& =r^{n-2} .
\end{aligned}
$$

The equality in (A.6) holds if and only if $L_{1}=L_{2} \cong C_{6} * K_{1}$ or $O_{1}(n-3), L_{3} \cong H_{0}(6) \uplus \frac{n-10}{2} K_{2}, H_{0}(8) \uplus$ $\frac{n-12}{2} K_{2}, H_{0}(10) \uplus \frac{n-14}{2} K_{2}, H_{1}(k) \uplus \frac{n-k-4}{2} K_{2}, H_{2}(k) \uplus \frac{n-k-4}{2} K_{2}$, or $H_{3}(k) \uplus \frac{n-k-4}{2} K_{2}$. If $L_{1}=L_{2} \cong C_{6} * K_{1}$, then $n \stackrel{2}{=} 10$ and $G \cong H_{0}(10)$. So $\operatorname{mi}(G)=\operatorname{mi}\left(H_{0}(10)\right)=16$, hence we get the extremal graph $H_{0}(10)$. If $L_{1}=L_{2} \cong O_{1}(n-3)$, then $n=8$ and $G \cong H_{0}(8)$. So $\operatorname{mi}(G)=\operatorname{mi}\left(H_{0}(8)\right)=8$, hence we get the extremal graph $H_{0}(8)$. Our result holds.

If $L_{3} \cong T_{1}(n-4), G$ is one of the graphs shown in Figs. 4(c), 4(d) and 4(f). By direct calculation, we have, for Fig. 4(c), $\operatorname{mi}(G)=13<16$; for Fig. $4\left(\right.$ d) $(n \geqslant 8), \operatorname{mi}(G)=3 r^{n-6}+2 \leqslant r^{n-2}$, the equality holds if and only if $n=8, G \cong H_{0}(8)$, hence we get extremal graph $H_{0}(8)$; for Fig. 4(f) $(n \geqslant 10)$, $\operatorname{mi}(G)=3 r^{n-6}+4 \leqslant r^{n-2}$, the equality holds if and only if $n=10, G \cong H_{0}(10)$. Hence, we assume $n_{1} \leqslant 2$, which implies $n_{1}=n_{2}=\cdots=n_{k}=2$. Since $G \supsetneqq B_{n}$, we conclude that $v_{1}$ must be adjacent to $v_{2}$. So $\operatorname{mi}(G)=r^{n-2}$ and $G \cong H_{1}(n)$.

This completes the proof. 